\title{
Severe Skin Toxicity Caused by Sequential Anti-PD-1 Antibody and Alectinib in Non-small-cell Lung Cancer: A Report of Two Cases and a Literature Review
}

\author{
Akihiro Nishiyama ${ }^{1,2}$, Yoshihiro Hattori ${ }^{3}$, Shinji Takeuchi ${ }^{1,2}$, Azusa Tanimoto ${ }^{1,2}$, \\ Miyako Satouchi ${ }^{3}$, Toshinori Murayama ${ }^{4}$ and Seiji Yano ${ }^{1,2}$
}

\begin{abstract}
:
Immune checkpoint inhibitors (ICIs) have demonstrated marked efficacy in some cancer patients, but they may cause various severe immune-related adverse events. Alectinib is a second-generation anaplastic lymphoma kinase (ALK)-tyrosine kinase inhibitor (TKI) approved for ALK-rearranged non-small-cell lung cancer (NSCLC). Alectinib is said to be safer than other TKIs. We conducted an investigator-initiated trial of alectinib, which also has RET kinase-inhibitory activity, against RET-rearranged NSCLC. Two RET-rearranged NSCLC patients experienced severe skin toxicity with alectinib after first undergoing anti-PD-1 antibody treatment with an ICI. These findings suggest that we should carefully follow patients for adverse effects of targeted drugs following ICI treatment.
\end{abstract}

Key words: severe skin toxicity, anti-PD-1 antibody, alectinib, ICIs

(Intern Med Advance Publication)

(DOI: 10.2169/internalmedicine.7472-21)

\section{Introduction}

Immune checkpoint inhibitors (ICIs), such as antiprogrammed death-1 (PD-1) antibody and anti-PD-ligand 1 (PD-L1) antibody, show anti-tumor efficacy by activating the immune system. ICIs have been approved for various types of tumors, including small-cell lung cancer (SCLC) and non-SCLC (NSCLC). While ICIs demonstrate marked efficacy in some patients, they may cause various severe immune-related adverse events (irAEs), including druginduced skin toxicity and interstitial lung disease (ILD), presumably by activating the autoimmune system. Previous studies have reported that severe irAEs, including ILD (1), were induced by a concurrent combination of ICIs and osimertinib, a third-generation epidermal growth factor receptor-tyrosine kinase inhibitor (EGFR-TKI) (2). However, severe adverse events associated with concurrent or sequential treatment with ICIs and other TKIs are not well re- ported.

Alectinib is a second-generation anaplastic lymphoma kinase (ALK)-TKI approved for ALK-rearranged NSCLC (3). Although several severe adverse events of alectinib, including dysgeusia, increased AST levels, increased bilirubin levels, rashes, and constipation, have been reported, their incidence is generally lower than those of other TKIs, such as crizotinib, an FDA-approved inhibitor for ALK and ROS1 kinases, and EGFR-TKIs (4-6).

We conducted an investigator-initiated trial of alectinib, which also has RET kinase-inhibitory activity, against RETrearranged NSCLC (UMIN000020628) (7). In the trial, two NSCLC patients with RET rearrangements experienced severe skin toxicity with alectinib administration after first having undergone anti-PD-1 antibody treatment. These findings suggest that we should carefully follow the patients for the adverse effects of targeted drugs following ICIs.

\footnotetext{
${ }^{1}$ Division of Medical Oncology, Cancer Research Institute, Kanazawa University, Japan, ${ }^{2}$ Cancer Center, Kanazawa University Hospital, Japan, ${ }^{3}$ Department of Thoracic Oncology, Hyogo Cancer Center, Japan and ${ }^{4}$ Innovative Clinical Research Center (iCREK), Kanazawa University Hospital, Japan

Received: March 3, 2021; Accepted: September 1, 2021; Advance Publication by J-STAGE: November 20, 2021

Correspondence to Dr. Akihiro Nishiyama, an0510@staff.kanazawa-u.ac.jp
} 


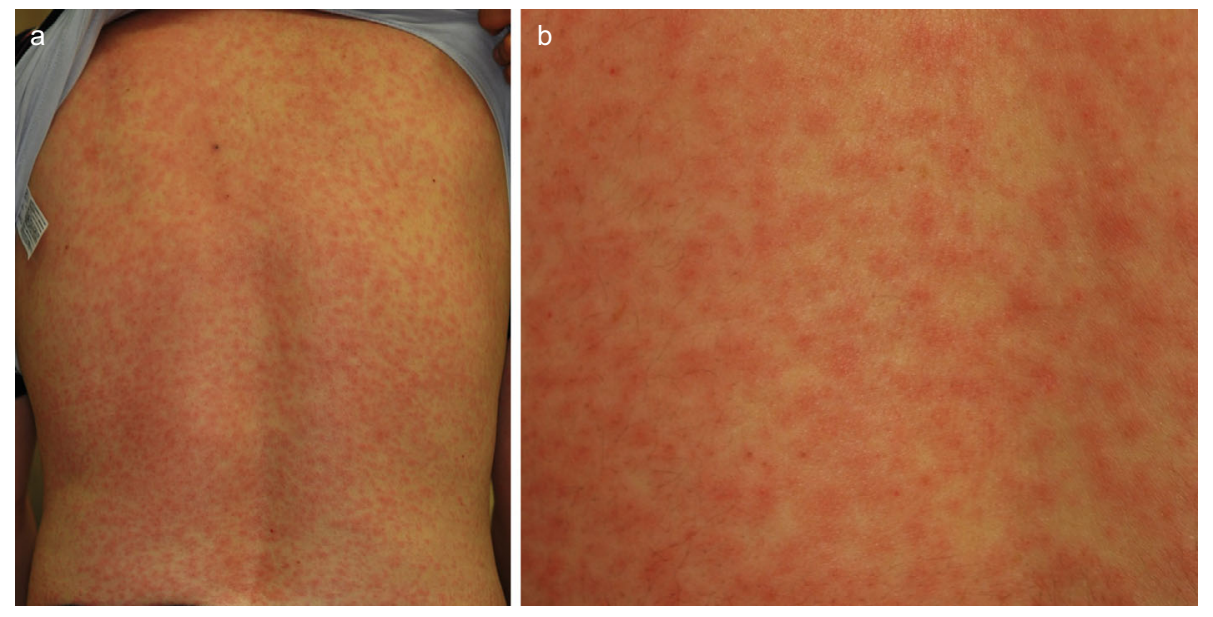

Figure 1. a: Skin rash observed in case 1. A woman in her 40s. Alectinib $600 \mathrm{mg}$ twice daily was started 24 days after the final administration of nivolumab. Picture shows erythema multiforme on day 13. b: Close-up picture of (a).

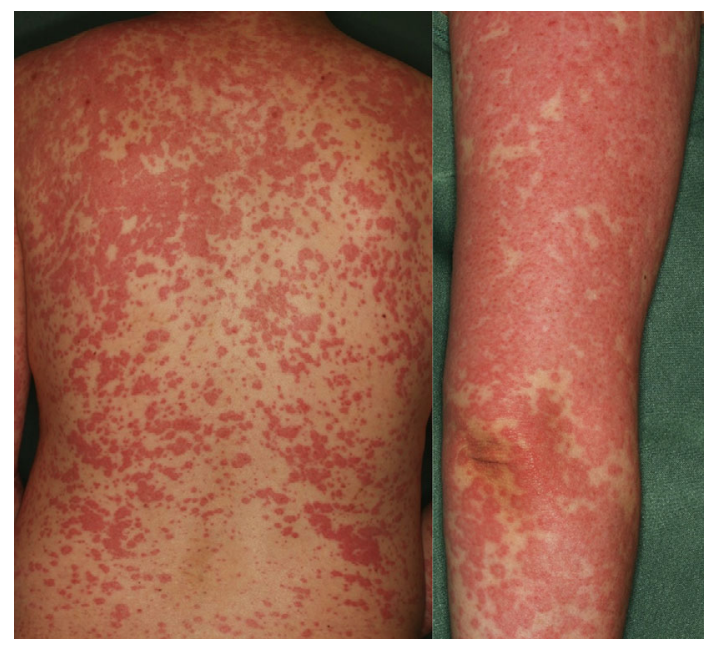

Figure 2. Skin rash observed in Case 2. A woman in her 30s. Alectinib $600 \mathrm{mg}$ twice daily was started eight weeks after the final administration of nivolumab. Picture shows erythema multiforme on day 12 .

\section{Case Reports}

\section{Case 1}

A woman in her 40s, a non-smoker, with a history of stage IIIB NSCLC (cT1aN3M0), was initially treated with concurrent chemotherapy (cisplatin and docetaxel) and radiation therapy. She had developed multiple metastases in the brain and bone that were controlled for eight years by multidisciplinary treatment, including stereotactic radiation and brain tumor resection. She showed disease progression with a primary lung tumor in the left upper lobe and was treated with conventional chemotherapy (second-line carboplatin+ pemetrexed, third-line docetaxel, fourth-line vinorelbine). As the fifth-line treatment, she received anti-PD-1 antibody nivolumab for six cycles. Nonetheless, her disease pro- gressed. Genome testing revealed a KIF5B-RET rearrangement, and she entered the Investigator-initiated Trial with alectinib (UMIN000020628).

Alectinib $600 \mathrm{mg}$ twice daily (FDA-approved dose for ALK-rearranged NSCLC) was started 24 days after the final administration of nivolumab. She presented with fever (38.0 ${ }^{\circ} \mathrm{C}$ : Grade 1) on day 10. Skin rash (erythema multiforme, Grade 3; Fig. 1a, b), increase in AST (Grade 3), and increased ALT (Grade 2) appeared on day 13. At that time, she had no mucosal lesion. Thus, alectinib was stopped, and $50 \mathrm{mg}$ prednisolone was administered intravenously for 7 days, followed by decreasing doses of oral prednisolone for 4 weeks. These symptoms, including rash, resolved by day 22.

\section{Case 2}

A woman in her 30s, a non-smoker, had a history of stage IV NSCLC (cTaN3M1b, OSS, HEP, PUL). She received seven lines of chemotherapy. As the eighth-line treatment, she received anti-PD-1 antibody nivolumab for four cycles. Nonetheless, her disease progressed. Genome testing revealed KIF5B-RET rearrangement, and she entered the Investigator-initiated Trial with alectinib (UMIN000020628).

Alectinib $600 \mathrm{mg}$ twice daily was started 8 weeks after the final administration of nivolumab. On day 5, she noticed that the right hypochondrial pain due to liver metastases decreased. However, she presented with a skin rash (Grade 2) on day 11 , and alectinib was discontinued. On day 12 , a severe skin rash and erythema multiforme were observed (Fig. 2), but she had no mucosal lesions. She was administered steroid pulse therapy of 1,000 mg methylprednisolone for 3 days. Her skin rash resolved by day 16 .

\section{Discussion}

Severe skin rashes (Grade 3) because of alectinib treatment are rare events $(0 \%-3 \%)(3,4)$. Table summarizes five 
Table. Literature Review of Patients with Severe Skin Rash Caused by Alectinib, Including Our Cases.

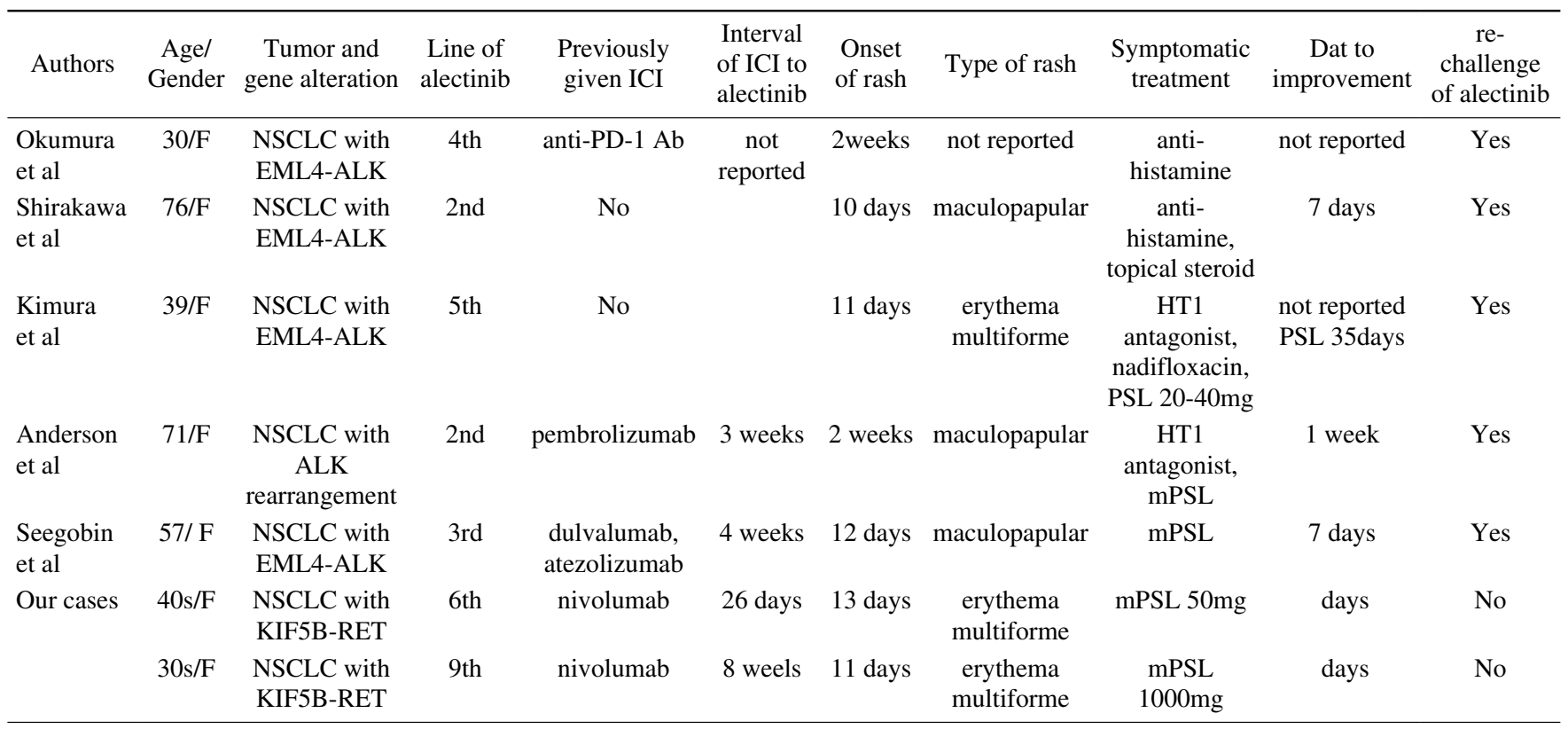

F: female, NSCLC: non-small cell lung cancer, EML4: echinoderm microtubule-associated protein-like4, ALK: anaplastic lymphoma kinase, KIF5: kinesin family member 5B, RET: rearranged during transfection, ICI; immune checkpoint inhibitor, HT1: hydroxytryptamine1, mPSL: methylprednisolone, PSL: prednisolone

case reports of severe skin rash caused by alectinib (8-12). Erythema multiforme caused by alectinib was reported in one case by Kimura et al. (8). In this case, erythema multiforme was observed on day 11 of alectinib treatment and improved by suspension of alectinib and introduction of a histamine-1 receptor antagonist, external preparation of nadifloxacin, and a medium-class steroid.

Severe skin rash (maculopapular) associated with sequential use of alectinib after ICI was reported in two cases $(9,10)$ (Table). As in the two cases reported here, erythema multiforme was observed after alectinib following treatment with anti-PD-1 antibody. This suggests that antiPD-1 antibody triggers serious skin toxicity upon alectinib treatment. The details of the mechanism are unknown, but it is possible that PD-1 inhibition activated the immune system and induced an immune response to alectinib. The intervals between nivolumab and alectinib treatments in our Cases 1 and 2 were one and two months, respectively.

Since previous reports showed that the onset of severe skin toxicity by alectinib treatment was 10-14 days with or without an ICI treatment history, particular attention is required during this period. In these case reports, the rash was improved by the suspension of alectinib and the addition of steroids with or without histamine-1 receptor antagonist, and the patients were re-challenged with a gradual increase in the dose of alectinib, which successfully controlled tumor progression. Our two cases with RET-rearrangement received alectinib as the protocol treatment in the Investigatorinitiated Trial, so alectinib was terminated at the onset of erythema multiforme, and patients were not re-challenged. Particularly in Case 2, we administered steroid pulse therapy because the severe erythema multiforme worsened, but the state of her lung cancer remained stable during steroid therapy.

The NCCN guidelines recommend TKI treatment for front-line treatment, as ICIs are not sufficiently effective in lung cancer with driver gene alterations. From the perspective of side effects, including serious skin toxicity, TKIs should be used on the front line, rather than ICIs, particularly in cases where TKIs might eventually be used.

The authors state that they have no Conflict of Interest (COI).

\section{References}

1. Ramos-Casals M, Brahmer JR, Callahan MK, Flores-Chávez A, Keegan N, Khamashta MA, et al. Immune-related adverse events of checkpoint inhibitors. Nat Rev Dis Primers 6: 38, 2020.

2. Schoenfeld AJ, Arbour KC, Rizvi H, Iqbal AN, Gadgeel SM, Girshman J, et al. Severe immune-related adverse events are common with sequential PD-(L)1 blockade and osimertinib. Ann Oncol 30: 839-844, 2019.

3. Hida T, Nokihara H, Kondo M, Kim YH, Azuma K, Seto T, et al. Alectinib versus crizotinib in patients with ALK-positive nonsmall-cell lung cancer (J-ALEX): an open-label, randomised phase 3 trial. Lancet 390: 29-39, 2017.

4. Peters S, Camidge DR, Shaw AT, Gadgeel S, Ahn JS, Kim DW, et al. Alectinib versus crizotinib in untreated ALK-positive nonsmall-cell lung cancer. N Engl J Med 377: 829-838, 2017.

5. Solomon BJ, Mok T, Kim DW, Wu YL, Nakagawa K, Mekhail T, et al. First-line crizotinib versus chemotherapy in ALK-positive lung cancer. N Engl J Med 371: 2167-2177, 2014.

6. Soria JC, Ohe Y, Vansteenkiste J, Reungwetwattana T, Chewaskulyong $\mathrm{B}$, Lee $\mathrm{KH}$, et al. Osimertinib in Untreated EGFR-Mutated Advanced Non-Small-Cell Lung Cancer. N Engl J Med 378: 113-125, 2018.

7. Takeuchi S, Yanagitani N, Seto T, Hattori Y, Ohashi K, Morise M, 
et al. Phase I/II study of alectinib in RET-rearranged previouslytreated non-small cell lung cancer (ALL-RET). Transl Lung Cancer Res 10: 314-325, 2021.

8. Kimura T, Sowa-Osako J, Nakai T, Ohyama A, Kawaguchi T, Tsuruta D, et al. Alectinib-Induced Erythema Multiforme and Successful Rechallenge with Alectinib in a Patient with Anaplastic Lymphoma Kinase-Rearranged Lung Cancer. Case Rep Oncol 9: 826-832, 2016.

9. Okuma Y, Tanaka Y, Kamei T, Hosomi Y, Okamura T. Alectinib for choroidal metastasis in a patient with crizotinib-resistant ALK rearranged positive non-small cell lung cancer. Onco Targets Ther 8: 1321-1325, 2015.

10. Seegobin K, Majeed U, Lou Y, Zhao Y, Manochakian R. Patients with high-grade alectinib-induced skin rash: How do we desensitize these patients? A case report and review of literature. SAGE
Open Med Case Rep 8: 2050313X20966895, 2020.

11. Shirasawa M, Kubotaa M, Harada S, Niwa H, Kusuhara S, Kasajima M, et al. Successful oral desensitization against skin rash induced by alectinib in a patient with anaplastic lymphoma kinasepositive lung adenocarcinoma: A case report. Lung Cancer 99: 6669, 2016.

12. Anderson BE, Luczak TS, Ries LM, Hoefs GE, Silva-Benedict AC. Successful alectinib desensitization in a patient with anaplastic lymphoma kinase-positive adenocarcinoma of the lung and alectinib-induced drug rash. J Oncol Pharm Pract 26: 2028-2030, 2020.

The Internal Medicine is an Open Access journal distributed under the Creative Commons Attribution-NonCommercial-NoDerivatives 4.0 International License. To view the details of this license, please visit (https://creativecommons.org/licenses/ by-nc-nd/4.0/).

(C) The Japanese Society of Internal Medicine Intern Med Advance Publication 